Iron oxide thin films prepared by ion beam induced chemical vapor deposition: Structural characterization by infrared spectroscopy

F. Yubero, M. Ocaña, and A. JustoL. ContrerasA. R. González-Elipe

Citation: Journal of Vacuum Science \& Technology A: Vacuum, Surfaces, and Films 18, 2244 (2000); doi: 10.1116/1.1286198

View online: http://dx.doi.org/10.1116/1.1286198

View Table of Contents: http://avs.scitation.org/toc/jva/18/5

Published by the American Vacuum Society

Contact Hiden Analytical for further details: w www.HidenAnalytical.com E info@hiden.co.uk CLICK TO VIEW our product catalogue

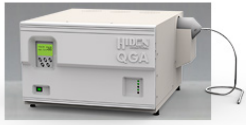

Gas Analysis

dynamic measurement of reaction gas stream catalysis and thermal analysis - molecular beam studies , dissolved species probes fermentation, environmental and ecological studies

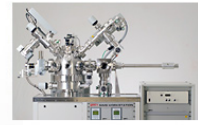

Surface Science , UHVTPD , SIMS

, end point detection in ion beam etch , elemental imaging - surface mappin

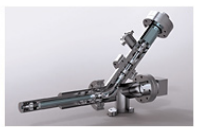

Plasma Diagnostics plasma source characterization etch and deposition process reaction , kinetic studies analysis of neutral and radical species

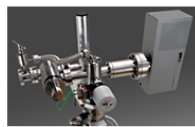

Vacuum Analysis partial pressure measurement and contro of process gases - reactive sputter process contro , vacuum diagnostics - vacuum coating process monitoring 


\title{
Iron oxide thin films prepared by ion beam induced chemical vapor deposition: Structural characterization by infrared spectroscopy
}

\author{
F. Yubero, ${ }^{\text {a) }}$ M. Ocaña, and A. Justo \\ Instituto de Ciencia de Materiales de Sevilla (CSIC-Universidad de Sevilla), C/ Américo Vespucio s/n, \\ E-41092 Sevilla, Spain \\ L. Contreras \\ Instituto de Investigaciones Químicas (CSIC-Universidad de Sevilla), C/ Américo Vespucio s/n, \\ E-41092 Sevilla, Spain
}

A. R. González-Elipe

Instituto de Ciencia de Materiales de Sevilla (CSIC-Universidad de Sevilla), C/ Américo Vespucio s/n, E-41092 Sevilla, Spain

(Received 2 September 1999; accepted 14 April 2000)

\begin{abstract}
Iron oxide thin films as hematite $\left(\alpha-\mathrm{Fe}_{2} \mathrm{O}_{3}\right)$ have been prepared by ion beam induced chemical vapor deposition. Very compact and dense films are obtained by this procedure. The thin films have been grown by bombardment of the substrate surfaces with $\mathrm{O}_{2}^{+}$ions or mixtures of $\mathrm{O}_{2}^{+}$and $\mathrm{Ar}^{+}$ ions, while a volatile precursor of iron [i.e., $\mathrm{Fe}(\mathrm{CO})_{5}$ ] is dosed onto the substrate surface. In the latter case, $\mathrm{Ar}$ atoms are incorporated within the iron oxide lattice. Atomic force microscopy, Rutherford backscattering spectroscopy, and x-ray photoelectron spectroscopy were utilized to characterize the films' surface morphology, stoichiometry and chemical state. The film structure has been analyzed by grazing angle x-ray diffraction (XRD) and infrared spectroscopies. In particular, infrared spectroscopy has permitted a thorough structural characterization of the films, even in the cases where XRD does not provide information about the structure. Thus, when $\mathrm{O}_{2}^{+}$ions are used for the synthesis, iron oxide thin films grow with a hematite structure with the $c$ axis of the crystallites perpendicular to the film surface. However, when an $\mathrm{Ar}^{+} / \mathrm{O}_{2}^{+}$ion mixture is used, the thin films have a hematite structure with the $c$ axis of the crystallites oriented parallel to the film surface. (C) 2000 American Vacuum Society. [S0734-2101(00)02605-1]
\end{abstract}

\section{INTRODUCTION}

Thin films of iron oxide have found many technological applications. Thus, iron oxide thin films as hematite $\left(\alpha-\mathrm{Fe}_{2} \mathrm{O}_{3}\right.$ ) attract interest as a gas sensor because they offer high sensitivity to some reducing gases without the application of noble metal catalyst. ${ }^{1}$ Besides, compact and flat iron oxide thin films of magnetite $\left(\mathrm{Fe}_{3} \mathrm{O}_{4}\right)$ or maghaemite $\left(\gamma-\mathrm{Fe}_{2} \mathrm{O}_{3}\right)$ are of technological interest in longitudinal magnetic recording media, owing to their magnetic ${ }^{2,3}$ and magneto-optical properties. ${ }^{4}$ Moreover, mixtures of iron oxide with other transition metal oxides are interesting due to their optical properties. ${ }^{5}$

For many of these applications, it is important to precisely control the surface morphology (i.e., density, roughness, manner of growth) and stoichiometry of the films. Several methods for preparing these thin films have been reported in the literature, including sputtering or plasma-assisted methods. ${ }^{6-8}$

In this article, we report on the preparation of iron oxide thin films by ion beam induced chemical vapor deposition (IBICVD). This method has been previously used in our laboratory for preparing other metal oxide and nitride thin films. ${ }^{9}$ A major characteristic of this method is the high densification and flatness of the prepared films. These are desir-

${ }^{a)}$ Electronic mail: yubero@cica.es able properties for thin films intended for optic and magnetooptic applications. ${ }^{4,5}$

The iron oxide thin films were analyzed by several spectroscopic techniques such as x-ray photoelectron spectroscopy (XPS) and Rutherford backscattering spectroscopy (RBS) to determine their surface and depth profile compositions. Grazing-angle x-ray diffraction (XRD) and infrared (IR) spectroscopy have been used for the structural characterization of the films. In order to characterize thin films, IR spectroscopy is typically used on a "fingerprint"' basis. However, a systematic analysis of IR spectra may also provide greater insight into the structure of the films. In particular, it is well known that IR spectra of a film contain information not only on the crystalline structure, but also on the orientation of the crystallographic axes with respect to the film surface. ${ }^{10-13}$

\section{EXPERIMENT}

Iron oxide thin films were prepared by IBICVD. This method consists of bombarding a suitable substrate with accelerated ions (e.g., $\mathrm{O}_{2}^{+}, \mathrm{Ar}^{+}, \mathrm{N}_{2}^{+}$) while flowing onto its surface a suitable precursor. ${ }^{9}$ In this article, flat silicon wafers, fused quartz, and $\mathrm{KBr}$ pellets were used as substrates for the different preparations. The volatile precursor used was $\mathrm{Fe}(\mathrm{CO})_{5}$ at partial pressures of $\sim 3 \times 10^{-5}$ Torr during preparation. Its decomposition at the substrate surface was done by $\mathrm{O}_{2}^{+} / \mathrm{Ar}^{+}$bombardment with a broad-source ion gun 
(HFQ 1303-3) with $400 \mathrm{eV}$ kinetic energy ions at a total pressure of $\sim 5 \times 10^{-4}$ Torr. Typical ion currents of $\sim 100 \mu \mathrm{A} / \mathrm{cm}^{2}$ were obtained at the sample position. Under these conditions, the growth rate of the iron oxide layers was approximately $\sim 1 \mathrm{~nm} / \mathrm{min}$.

Topographic images of the iron oxide films deposited on the fused quartz and Si wafers were taken with a TMX Explorer atomic force microscope (AFM) from Topometrix with a $\mathrm{Si}_{3} \mathrm{~N}_{4}$ tip working in contact mode.

RBS measurements on the iron oxide thin films deposited on the silicon wafers were carried out in the ion accelerator ARAMIS (Orsay, France) ${ }^{14}$ using $2.4 \mathrm{MeV} \alpha^{++}$particles. The experimental RBS spectra were simulated using the RUMP code. ${ }^{15}$

XPS spectra were acquired in an ESCALAB210 electron spectrometer working at constant pass energy mode. The spectra were excited with $\mathrm{Al} K_{\alpha}$ radiation. The energy resolution was such that the full width at half maximum of a $\mathrm{Ag}$ $3 d_{5 / 2}$ photoemitted peak of pure silver sample was $1.1 \mathrm{eV}$. The reference of the binding energy scale was taken at the $\mathrm{C} 1 \mathrm{~s}$ peak of the carbon contaminating the surface of the samples at a value of $284.6 \mathrm{eV}$.

Grazing-angle XRD spectra were acquired in a Siemens D5000 diffractometer at an incidence angle of $0.5^{\circ}$.

IR absorption spectra were recorded in the transmission mode in a Nicolet 510 Fourier transform infrared spectrometer. For the IR analysis, the iron oxide thin films were deposited on $\mathrm{KBr}$ pellets to ensure the maximum transmission and high quality of the spectra. Similar spectra, although with lesser quality, were obtained when silicon wafers were used as substrates in simultaneous preparations.

In this article, the different substrates (i.e., $\mathrm{Si}, \mathrm{KBr}$, and quartz) were chosen to be able to characterize the iron oxide thin films with the different techniques (i.e., IR, AFM, RBS, XRD, and XPS). No significant effect was detected in the characteristics of the iron oxide thin films produced by IBICVD by changing the substrates. So, the main agent that determines the structure of the films is that they grow under ion bombardment.

\section{RESULTS AND DISCUSSION}

A characteristic feature of the IBICVD technique is that it produces very compact, flat and dense films, with a practical absence of voids and pores. A typical image of the surface topography of an iron oxide thin film prepared on a silicon substrate by this technique is shown in Fig. 1. It shows that the surface roughness is very low (note that the vertical scale is $0-2 \mathrm{~nm}$ for an area of $1 \mu \mathrm{m} \times 1 \mu \mathrm{m}$ scanned by the microscope), which stresses the surface flatness of the sample. This is a general result obtained from thin films prepared by this technique, independent of the ion species $\left(\mathrm{O}_{2}^{+}\right.$, or mixtures $\mathrm{O}_{2}^{+} / \mathrm{Ar}^{+}$) used for the preparations.

The stoichiometry and chemical composition of the deposited films were studied by XPS and RBS spectroscopies. The photoemission spectra of the prepared thin films of iron oxide show that the sample surface mainly consists of $\mathrm{O}$ and $\mathrm{Fe}$ in a stoichiometric ratio close to that of $\mathrm{Fe}_{2} \mathrm{O}_{3}$ (within

\section{$\mathrm{Fe}_{2} \mathrm{O}_{3}$ deposited on fused quartz by IBICVD}

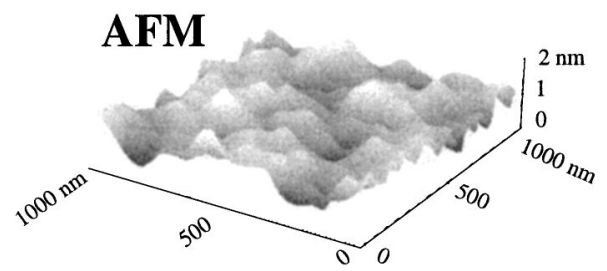

FIG. 1. AFM image of the surface of a typical iron oxide thin film deposited by IBICVD on fused quartz.

5\%). The Fe $2 p$ photoelectron spectrum (see Fig. 2) of all the samples is characterized by the spin orbit $\mathrm{Fe} 2 p$ doublet at $711.0 \mathrm{eV}$ binding energy (for the $\mathrm{Fe} 2 p_{3 / 2}$ peak) and a characteristic satellite at $8 \mathrm{eV}$ higher binding energy. This type of spectra is typical of $\alpha-\mathrm{Fe}_{2} \mathrm{O}_{3}$, as it has been previously reported. ${ }^{16}$ XPS also showed that the surface of the sample was contaminated by carbon due to manipulation in air. In fact, this carbon disappeared to levels of $\sim 2$ at. \% after a mild bombardment with $\mathrm{O}_{2}^{+}(500 \mathrm{eV})$. Note that although there is much carbon in the molecule of the precursor of iron used for the preparation, it is preferentially sputtered out by the $\mathrm{O}_{2}^{+}$ions during the growth, so very little carbon is incorporated in the film as contaminant.

The depth profile composition and film thicknesses were determined by RBS. Figure 3 shows spectra of two iron oxide thin films prepared with (a) $\mathrm{O}_{2}^{+}$or (b) a mixture $\mathrm{O}_{2}^{+} / \mathrm{Ar}^{+}$ $\left(90 \% \mathrm{Ar}\right.$ and $\left.10 \% \mathrm{O}_{2}\right)$ on a $\mathrm{Si}$ substrate. Simulation of the RBS experimental spectra by means of the RUMP program yields a film composition of $\mathrm{FeO}_{1.5 \pm 0.1}$ in the two cases. The spectra also confirm the sharp character of the substrate/film interface, within the resolution limits of the technique (i.e., $\sim 10 \mathrm{~nm}$ ). The thicknesses of these two films as determined by this analysis were 140 and $90 \mathrm{~nm}$, respectively, assuming a density of $5.0 \mathrm{~g} / \mathrm{cm}^{3}$ for the iron oxide films. This range of thicknesses is typical of all the films studied in this article. It is also apparent that in the films prepared with a mixture of $\mathrm{O}_{2}^{+}$and $\mathrm{Ar}^{+}$ions, $\sim 4$ at. $\%$ of $\mathrm{Ar}$ atoms becomes homogeneously incorporated within the whole film thickness. Annealing these films in air at $500{ }^{\circ} \mathrm{C}$, does not modify the spectrum of Fig. 3(b). This result indicates that the Ar atoms are homogeneously distributed in lattice vacancies of the

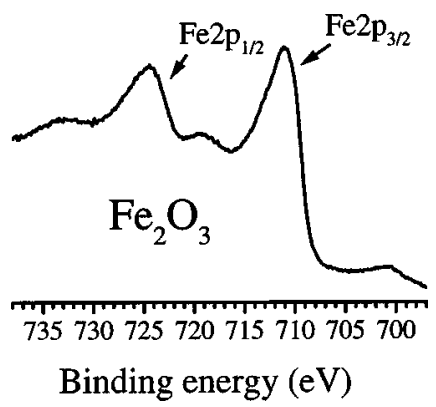

FIG. 2. Fe $2 p$ core level photoelectron spectrum of a typical iron oxide thin film prepared by IBICVD. 


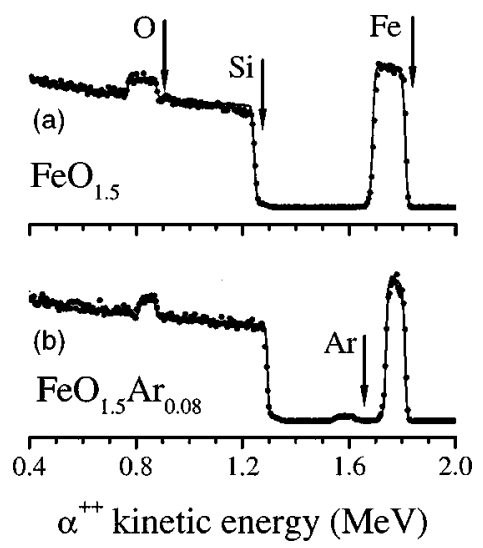

FIG. 3. RBS spectra of iron oxide thin films prepared by IBICVD by using (a) $\mathrm{O}_{2}^{+}$or (b) $\mathrm{O}_{2}^{+} / \mathrm{Ar}^{+}$ions. Dots: raw data. Solid lines: RUMP simulations.

film through the whole film thickness. The high compaction of the films prepared by IBICVD must also be claimed to explain that no Ar atoms are released, even after annealing at $500^{\circ} \mathrm{C}$.

A common characteristic of most of the thin films of the materials synthesized by IBICVD is their low crystallinity, as determined by XRD. This feature has also been observed for the $\mathrm{Fe}_{2} \mathrm{O}_{3}$ thin films prepared in this work. This is illustrated in Fig. 4, which shows a set of XRD plots for $\mathrm{Fe}_{2} \mathrm{O}_{3}$ thin films grown under different conditions. Thus, in this figure, samples A and B were prepared by $\mathrm{O}_{2}^{+}$bombardment, whereas sample $\mathrm{C}$ was prepared by bombardment with a $\mathrm{Ar}^{+} / \mathrm{O}_{2}^{+}$ion mixture $\left(90\right.$ mole $\% \mathrm{Ar}$ and 10 mole $\% \mathrm{O}_{2}$ ). The thicknesses of these samples are 300,50 , and $70 \mathrm{~nm}$ for samples A, B, and C, respectively. These samples have been selected as representative of the structural behavior of the $\mathrm{Fe}_{2} \mathrm{O}_{3}$ samples prepared by IBICVD, which can be summarized as follows:

(i) The XRD spectra of films with a thickness larger than $\sim 100 \mathrm{~nm}$ (as sample A in Fig. 4) depict the typical peaks of the hematite $\alpha-\mathrm{Fe}_{2} \mathrm{O}_{3}$ structure, although with a relative intensity that differs from that of a randomly oriented polycrystalline sample. This can be seen in Fig. 4, when compar-

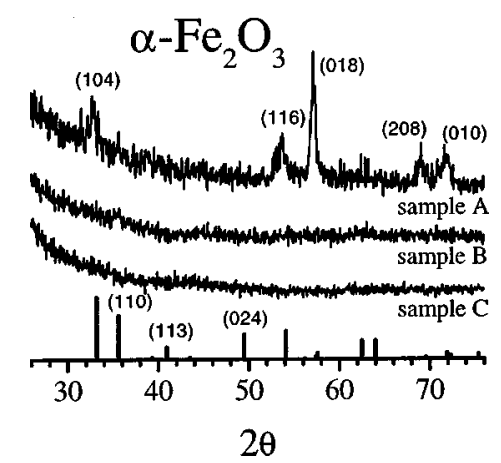

FIG. 4. XRD plots of iron oxide thin films prepared by IBICVD. Samples A and $\mathrm{B}$ were prepared by $\mathrm{O}_{2}$ bombardment, whereas sample $\mathrm{C}$ was prepared by bombardment with a $\mathrm{Ar}^{+} / \mathrm{O}_{2}^{+}$mixture $\left(90 \% \mathrm{Ar}\right.$ and $\left.10 \% \mathrm{O}_{2}\right)$. The thicknesses of these samples are 300,50, and $70 \mathrm{~nm}$ for samples A, B, and C, respectively.

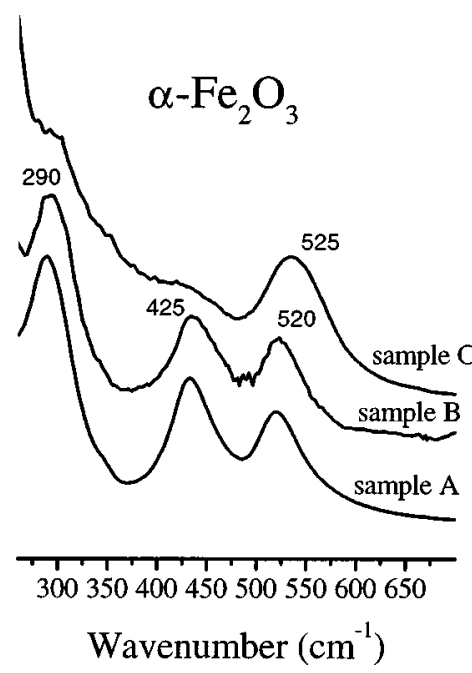

FIG. 5. IR spectra of iron oxide thin films prepared by IBICVD. The spectra correspond to the same samples characterized by XRD in Fig. 4.

ing both the diagram of sample $\mathrm{A}$ and the theoretical intensities of the hematite $\alpha-\mathrm{Fe}_{2} \mathrm{O}_{3}$ structure (vertical lines), which reveals a preferential orientation of some crystallographic planes.

(ii) For samples of lower thickness $(\leqslant 70 \mathrm{~nm}$, as samples $\mathrm{B}$ and $\mathrm{C}$ in Fig. 4), the XRD spectra do not depict welldefined peaks. This suggests that either the films do not present a long-distance order or that, for very thin films, the XRD peak intensities are so low that they cannot be detected under our experimental conditions.

It is well known that XRD only provides structural information of materials where there is a well-defined order at long distances. For thin films as those in Fig. 4, where no well-defined peaks could be recorded by XRD, IR spectroscopy can be an alternative probe for their structural characterization. The basic theoretical principles for this structural analysis of thin films can be found in articles published in the 1960 's. ${ }^{10-12}$

It has been amply reported that only transverse vibrational modes can be detected for thin films when the IR absorption spectra are recorded at normal incidence of the radiation. Under these conditions, the electric field vector of the radiation is parallel to the substrate surface. Consequently, not all transverse modes of a given anisotropic solid are observed, but rather, only those corresponding to lattice vibrations parallel to the film surface. Therefore, the relative intensity observed for transverse modes associated with each crystallographic direction can be used to obtain information on the orientation of the crystal axes in the film.

The IR absorption spectra measured at normal incidence of the selected films are shown in Fig. 5. The IR spectra of samples A and B are quite similar. They represent three intense bands at 290,435 , and $520 \mathrm{~cm}^{-1}$ that can be related to $E_{u}$ vibrational modes (electric field $E \perp c$ ) of hematite (see Table I). It should be noted that although the $A_{2 u}$ modes $\left(E \|_{c}\right)$ of hematite appear at frequencies close to those of two $E_{u}$ modes (290 and $520 \mathrm{~cm}^{-1}$ ), the relative intensity of the bands observed for samples $\mathrm{A}$ and $\mathrm{B}$ is consistent with that 
TABLE I. IR transverse modes for $\alpha-\mathrm{Fe}_{2} \mathrm{O}_{3}$ taken from Ref. 18 .

\begin{tabular}{cc}
\hline \hline Symmetry & $v_{T}\left(\mathrm{~cm}^{-1}\right)$ \\
\hline$E_{u}(E \perp c)$ & 524 \\
& 437 \\
& 286 \\
$\mathrm{~A}_{2 u}(E \| c)$ & 227 \\
& 526 \\
& 299 \\
\hline \hline
\end{tabular}

expected for the $E_{u}$ modes. ${ }^{17}$ This suggests that the $A_{2 u}$ modes do not contribute to these spectra. The presence of the $E_{u}$ modes in the absorption spectrum of the film indicates that for samples A and B, the crystallographic $c$ axis of the deposited hematite is perpendicular to the film surface. It should be noted that although sample B does not show any long-range order by XRD (see Fig. 4), its IR spectrum is similar to that of sample A, indicating that their crystal structure and orientation are also similar.

In the IR spectrum of sample $\mathrm{C}$, only an intense band at $525 \mathrm{~cm}^{-1}$ along with a weak feature at $\sim 290 \mathrm{~cm}^{-1}$ are present, whereas the $E_{u}$ mode at $435 \mathrm{~cm}^{-1}$ has almost no intensity. Therefore, the bands observed for sample $\mathrm{C}$ can be mainly attributed to the transverse $A_{2 u}$ modes of hematite (see Table I). This phase could not be detected by XRD (see Fig. 4), probably due to the small thickness of the film or the low crystallinity of the sample. Note that XRD has a lower sensitivity to short-range order than IR spectroscopy. The presence in the spectrum of the film of only $A_{2 u}$ modes also indicates that the crystallographic $c$ axis of the deposited hematite must be oriented preferentially parallel to the film surface, because such modes vibrate parallel to this axis.

To confirm the preferential crystallographic orientation of the $c$ axis normal to the film surface of sample A, transmission IR spectra were also acquired, varying the IR radiation angle of incidence, $\theta$, with respect to the surface normal. The results are shown in Fig. 6. Note that the $E_{u}$ longitudinal

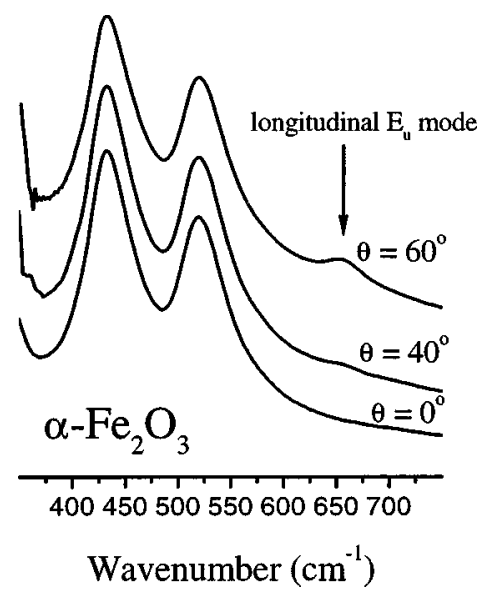

FIG. 6. Effect of varying the IR radiation angle of incidence, $\theta$, with respect to the surface normal. The IR spectra correspond to sample A in Figs. 4 and 5 . mode appears in the spectrum at $\sim 660 \mathrm{~cm}^{-1}$ when the sample is tilted, as expected for a preferential orientation of the $c$ axis normal to the surface. ${ }^{18}$

So, samples $\mathrm{B}$ and $\mathrm{C}$, which present flat patterns to $\mathrm{XRD}$, show $\alpha-\mathrm{Fe}_{2} \mathrm{O}_{3}$ crystalline structure with different crystal orientations, as determined by IR spectroscopy. Note that sample $\mathrm{B}$ was prepared by $\mathrm{O}_{2}^{+}$bombardment, while for sample $\mathrm{C}$ a mixture of $\mathrm{O}_{2}^{+}$and $\mathrm{Ar}^{+}$was used. Therefore, different deposition conditions of the iron oxide thin films induce the growth of different crystallographic planes.

Finally, we emphasized that our results show that it is possible to prepare very thin films (thickness $\leqslant 100 \mathrm{~nm}$ ) of $\alpha-\mathrm{Fe}_{2} \mathrm{O}_{3}$ by IBICVD, controlling the crystallographic orientation by varying the preparation protocol. Moreover, a thorough IR characterization identifies the crystallographic orientation of the deposited species

\section{CONCLUSIONS}

In this article, we have shown that very compact and smooth thin films having an $\mathrm{Fe}_{2} \mathrm{O}_{3}$ stoichiometry can be prepared by IBICVD, as shown by AFM, XPS, RBS, and IR. Although all the films presented similar surface morphology (i.e., flat, dense, compact), their structural characteristics were highly dependent on the preparation protocol. Thus, by using mixtures of $\mathrm{Ar}^{+}$and $\mathrm{O}_{2}^{+}$ions for the preparation, poorly ordered structures were obtained where a $\sim 4$ at. $\%$ of Ar atoms became incorporated into the lattice vacancies of the iron oxide films. For these samples, IR spectroscopy shows that the thin films have a local hematite structure with the $c$ axis of the crystallites parallel to the film surface. When only $\mathrm{O}_{2}^{+}$ions are used for the synthesis, XRD analysis of samples with a thickness greater than $\sim 100 \mathrm{~nm}$ shows the typical peaks of hematite. However, either no peaks or very small peaks could be observed in XRD for very thin samples (thickness $\leqslant 70 \mathrm{~nm}$ ). In spite of this, IR spectroscopy showed that, in this case, the iron oxide thin films grow with a hematite structure with the $c$ axis of the crystallites oriented perpendicular to the film surface.

\section{ACKNOWLEDGMENTS}

The authors thank the CICYT (Project No. MAT97-0689) and NATO (Project No. CRG 970200) for financial support. They also acknowledge D. Zanghi, C. Clerk, A. Traverse, and the staff at ARAMIS for their assistance in the RBS measurements.

\footnotetext{
${ }^{1}$ A. A. Vasiliev, Sens. Actuators B 7, 626 (1992).

${ }^{2}$ D. V. Dimitrov, G. C. Hadjipanayis, V. Papaefthymion, and A. Simopoulos, J. Vac. Sci. Technol. A 15, 1473 (1997).

${ }^{3} \mathrm{R}$. M. Cornel and U. Schwertmann, The Iron Oxides $(\mathrm{VCH}$, New York, 1996).

${ }^{4} \mathrm{H}$. Guerrero et al., Nanospace 98, 2nd NASA Conference on Nano/ Microtechnology for Space Applications, Houston, TX, Nov. 1-6, 1998. ${ }^{5}$ P. R. Athey, M. F. Tabet, and F. K. Urban, J. Vac. Sci. Technol. A 15, 998 (1997).

${ }^{6}$ D. T. Margulis, F. T. Parker, F. E. Spada, R. S. Goldman, J. Li, R.
} 
Sinclair, and A. E. Berkowitz, Phys. Rev. B 53, 9175 (1996).

${ }^{7}$ Y. K. Kim and M. Oliveria, J. Appl. Phys. 75, 431 (1994).

${ }^{8}$ Y. K. Kim, Y. Guo, and S. A. Chambers, Surf. Sci. 371, 358 (1997).

${ }^{9}$ J. P. Espinós, A. Fernández, A. Caballero, V. M. Jiménez, J. C. Sánchez-

López, , L. Contreras, D. Leinen, and A. R. González-Elipe, Adv. Mater.,

Chem. Vap. Deposition 3, 219 (1997).

${ }^{10}$ D. W. Berreman, Phys. Rev. 130, 2193 (1963).

${ }^{11}$ G. L. Bottger and A. L. Geddes, J. Chem. Phys. 46, 3000 (1967).
${ }^{12}$ F. Proix and M. Balkanski, Phys. Status Solidi 32, 119 (1969).

${ }^{13}$ J. S. Ahn, H. S. Choi, and T. W. Noh, Phys. Rev. B 53, 10310 (1996).

${ }^{14}$ H. Bernas et al., Nucl. Instrum. Methods Phys. Res. B 62, 146 (1992).

${ }^{15}$ L. R. Doolittle, Nucl. Instrum. Methods Phys. Res. B 9, 344 (1985).

${ }^{16}$ W. Weiss, Surf. Sci. 377/379, 943 (1997).

${ }^{17}$ C. J. Serna, J. L. Rendon, and J. E. Iglesias, Spectrochim. Acta A 38, 797 (1982).

${ }^{18}$ S. Onari, T. Arai, and K. Kudo, Phys. Rev. B 16, 1717 (1997). 